\title{
Ciclos de vida biológicos y culturales, una propuesta integradora
}

Biological and cultural life cycles, an integrating proposal

\section{REsUMEN}

Presentamos aquí un intento de síntesis teórica de las relaciones dinámicas entre los ciclos de vida biológicos y los ciclos de vida culturales. Para ello nos basamos en los trabajos del profesor Juan Samaja y su esquema sobre el estudio del proceso de salud-enfermedad-atención. Básicamente el planteamiento se origina en los trabajos de Bateson y el pensamiento complejo sobre los tipos lógicos y en los conceptos dialécticos de supresión-conservación-superación. En este artículo aplicamos esos conceptos a los ciclos biológicos y a los ciclos culturales, a su relación desde un punto de vista antropológico. En síntesis, la idea es ir construyendo un marco de comprensión para el análisis del vínculo entre la naturaleza y la cultura. Este trabajo es una primera exploración en ese sentido; nuestro objetivo es encontrar algunas reglas que permitan comprender las relaciones que se establecen entre ambos ámbitos.

Palabras clave: naturaleza, cultura, complejidad, antropología.
Diego Díaz-Córdova

Doctor en Ciencias

Antropológicas. Profesor de

Antropología, Universidad

Nacional de Lanús.

Departamento de Salud

Comunitaria, Universidad

de Buenos Aires, Grupo

Antropocaos, Argentina.

$\square$ didibart@gmail.com

(1) ORCID: 0000-0002-1524-3529

$\triangle$ Google Scholar 


\begin{abstract}
An attempt of a theoretical synthesis of the dynamic relationships between biological lifecycles and cultural lifecycles is presented in this text. For this purpose, we ground our research in the works of professor Juan Samaja and his outline on the study of the health-disease-care process. Basically, the approach originates in Bateson's work and complex thinking about logical types and from the dialectical concepts of suppression-conservation-overcoming. These concepts are applied in this text to both biological and cultural cycles from an anthropological perspective. In short, the idea is to build a framework of understanding for the analysis of the link between nature and culture. This work is a first exploration in that sense. The objective is to find some rules that help understanding the relationships established between both areas.
\end{abstract}

Keywords: nature, culture, complexity, anthropology.

\title{
Introducción
}

i algo queda claro en el proceso de comprender qué es el Homo sapiens, es que la cultura y la biología se encuentran imbricadas; no hay una separación precisa y cuando la hay es simplemente un artificio (dicho esto en el mejor de los sentidos) que solo tiene efectos a nivel analítico.

El Homo sapiens hace su aparición en la Tierra ya cargado de cultura. Claramente, los homínidos que nos precedieron poseían fuertes rasgos culturales, que se manifiestan en el registro arqueológico, en forma de uso del fuego, de uso de herramientas y aún en expresiones artísticas o cuando menos, en apuestas estéticas. Pero esa cultura (que con el tiempo fue haciéndose cada vez más central hasta llegar a ser indispensable para nuestra supervivencia) se encuentra mezclada con la propia dotación biológica (Aiello y Wheeler, 1995; Binford, 1991). El caso extremo, seguramente, pueda referirse a los organismos modificados genéticamente (transgénicos) que consumimos a diario en nuestros "alimentos", en forma directa o indirecta. Ya no sólo podemos modificar la naturaleza por selección artificial de rasgos fenotípicos; hoy día podemos cambiar el propio ADN, aunque como con el personaje de la novela de Mary Shelley, no tengamos muy claros cuáles pueden ser los efectos de tamaña modificación. Hay, entonces, evidentes causas biológicas que producen cambios culturales y hay causas culturales que inducen cambios biológicos. Y en esa maraña, simplemente somos. 
Hablar de ciclos de vida implica necesariamente meterse en un territorio complicado, asumir que las categorías con que intentamos explicarlos, no van a dar cuenta de la complejidad de los casos, que vamos a tener que lidiar con fenómenos ambiguos en cuanto a su posible origen y que habrá que tomar decisiones metodológicas y teóricas no necesariamente probadas o aceptadas en su mayoría. Y es que los ciclos de vida tienen un impacto en los aspectos señalados, se afectan mutuamente y si bien se podría hablar (en teoría) de un ciclo de vida biológico, si asumimos la hipótesis de que el Homo sapiens es cultura y biología todo junto, entonces no puede existir en forma aislada, salvo, claro está, a efectos analíticos.

Los ciclos de vida, sean biológicos o culturales, pueden definirse a partir de la aparición u ocurrencia de determinados eventos; lo suficientemente visibles y que acarrean, a partir de su surgimiento, cambios determinados tanto en el cuerpo como en la conducta de los individuos. Si bien la vida es un continuum y no existe un cambio de etapa que sea cualitativamente claro, sí existe un cúmulo de evidencias que permiten encontrar categorías diferenciadas entre etapas diferentes. En definitiva, de lo que se trata es de aplicar una estrategia analítica, que permita tener una clasificación lo suficientemente rigurosa y evidente como para que otro pueda replicarla.

Nuestra hipótesis de partida será la de que los ciclos biológicos del Homo sapiens son universales, se dan en todas las sociedades, más allá de las diferencias individuales. Tendrán una expresión, en este sentido, claramente estadística. Por el contrario, los ciclos de vida culturales dependerán de las circunstancias particulares de cada sociedad, de acuerdo con sus intereses y a las restricciones materiales y ecológicas en las que se encuentran inmersas. Su expresión será, en todo caso, cualitativa. Por otra parte, los ciclos biológicos vienen acompañando al ser humano desde su aparición hace 300.000 años (Roberts, 2010), con cambios dados únicamente en cuanto a la aceleración o al retardo de la aparición del evento que marca su comienzo o fin. Los ciclos de vida culturales son más dinámicos, sometidos a otro tipo de régimen, en el sentido de que permitirían no solo atrasar o retardar la aparición de los eventos, sino incluso modificarlos, suprimirlos o agregarlos, de acuerdo con las circunstancias en las que se encuentre.

La idea general que guía este artículo se funda en la intención de ver cómo se coordinan datos de diferente naturaleza, tanto los biológicos como los culturales; de qué manera se pueden poner en un plano de coherencia que permita comprender mejor la posición que ocupa el desarrollo 
humano; y proponer un ejemplo que brinde coherencia partiendo de marcos teóricos diferentes (el biológico y el cultural) y aún metodologías también diferentes (cuantitativas y cualitativas).

En este sentido echaremos mano de los trabajos del profesor Juan Samaja que muestran cómo pueden vincularse diferentes niveles de análisis. En sus trabajos sobre una "Epistemología de la Salud" (Samaja y Galende, 2009), aborda la problemática aquí planteada, asumiendo un conjunto de categorías autonómicas interconectadas en una espiral jerárquica. Jerárquica en un sentido lógico y no axiológico. Sus planteos teóricos acerca de los diferentes tipos de ordenamiento, desde el molecular, hasta el simbólico-cultural, nos muestran los límites de las "totalidades organizadas" (esas estructuras autonómicas denominadas como el "diagrama de la epigenética"). Pero a la vez nos muestran los vínculos teóricos entre esas entidades, a partir de los conceptos de "supresión", "conservación" y "superación", tributarios, claro está, de los estudios de Hegel y del concepto "recaída en la inmediatez" (Hegel, 2013).

El texto comienza repasando los conceptos samajianos, para establecer desde el principio el método que vamos a seguir, en nuestro intento de poner en un marco coherente los vínculos entre ambos ciclos. Luego seguiremos definiendo los ciclos de vida biológicos, tal como los propone el antropólogo Barry Bogin en su libro "Patrones del crecimiento humano" (Bogin, 1999). A continuación, pasaremos revista a cómo distintas culturas proponen ciclos de vida culturales, diferentes en cada una de ellas, intentando desentrañar las motivaciones y condiciones para esos cambios. Por último, intentaremos realizar una síntesis entre ambas posturas, utilizando ahora sí los conceptos samajianos, intentando proponer una solución coherente al problema del crecimiento biológico y cultural de los seres humanos.

Una última aclaración. Este artículo es un texto básicamente teórico y analítico, por lo tanto, la separación que se propone se corresponde pura y exclusivamente con estos dos objetivos mencionados. La realidad es, en definitiva, una totalidad indiferenciada y es sólo a efectos de conocer esa realidad, que nosotros los humanos, en un proceso cognitivo de abstracción, podemos dividirla. Esa división no es arbitraria, aun cuando no seamos conscientes de ello. Responde siempre a un marco teórico, a una posición frente al mundo, a una manera de ver lo que nos rodea. Y este caso no es la excepción. Todo lo contrario, la división entre ambos ciclos ocurre debido a nuestra insistencia en una forma particular de comprender a la propia antropología. Aquella que plantea que la realidad es tan compleja que vale la pena no dejar nada en el tintero. En todo caso las omisiones o errores 
que pudieran aparecer se deben pura y exclusivamente a nuestra propia ignorancia e incapacidad; advertidos ya, pueden continuar con la lectura.

\section{Un modelo para la comprensión de las totalidades}

Juan Samaja fue uno de los más importantes epistemólogos y metodólogos de la Argentina. A su lucidez como filósofo, necesaria para una reflexión acertada sobre el proceso de conocimiento, sumaba su experiencia en el trabajo empírico. A diferencia de muchos epistemólogos de renombre, que sólo analizan lo que otros hacen, Samaja tenía una vasta experiencia en investigación concreta y eso lo colocaba en una clara posición ventajosa. En el texto que invocamos en este artículo, el autor intenta (y creemos que logra) establecer los vínculos entre los diferentes niveles que conforman los procesos de salud y enfermedad. Se parte de la idea de que estos procesos no afectan ni son afectados únicamente por circunstancias que podemos denominar "biológicas", sino que están atravesadas por todas las determinaciones de la vida humana.

Su postura se aleja del naturalismo positivista, tanto como del constructivismo irracional; ni entiende a los fenómenos como una pura objetividad, ni los clasifica según una caprichosa subjetividad. No hay una sola verdad, pero tampoco impera un relativismo disolvente. Este posicionarse en un punto medio (¿aristotélico?), lo lleva directo a pensar en los fenómenos desde una perspectiva basada en la complejidad. Un sistema complejo requiere de una organización dinámica, que debe estar sometida a alguna clase de retroalimentación (Morin, 2009).

El pensamiento complejo no es una unidad monolítica, en donde todos los que se asumen como parte de este, comparten marcos filosóficos, epistemológicos, teóricos o metodológicos. Las diferencias son inmensas (Reynoso, 2009); pero como este no es un artículo sobre esa clase de "pensamiento" (simplemente lo usamos como herramienta), vamos a tomar algunos marcadores en común, como para que el lector pueda tener una idea acerca del tema. Rápidamente podemos decir que el pensamiento complejo surge a mediados de los años 40, de la mano de la "teoría general de sistemas" (Bertalanffy, 2018) y la cibernética (Ashby, 2015), como un intento de unificar las ciencias bajo un nuevo paradigma que se aparte del reduccionismo, así como de los trabajos sobre la nueva ciencia de la computación que realizó von Neumann (Aspray, 1993).

De hecho, en el trabajo de von Neumann sobre "autómatas celulares", la pregunta que hizo el sabio húngaro fue la de si era posible que una máquina computacional teórica pudiera reproducirse (y conservar 
el mismo grado de complejidad) de la misma forma en la que los organismos biológicos lo hacen. La cultura imitando la biología. Pero más allá de estas particularidades, el pensamiento complejo se caracteriza por entender a los fenómenos desde una perspectiva que contempla una serie de propiedades como la retroalimentación (la posibilidad de un cambio endógeno a partir de señales que son interpretadas internamente y en donde el sistema reacciona en consecuencia), la morfogénesis (la posibilidad de los sistemas de generar nuevas formas a partir de los elementos existentes), la homeostasis (la capacidad de un sistema de autorregularse) y las propiedades emergentes (las propiedades que exhiben los sistemas y que no pueden ser deducidas de las relaciones lineales de sus partes componentes). Son sistemas dinámicos y propensos tanto al cambio como la estabilidad (Reynoso, 1998). La clave en el "pensamiento complejo" está en pensar en totalidades, atendiendo a la interacción entre sus partes.

En su libro "Epistemología de la salud", Samaja plantea los vínculos entre los diferentes tipos de reproducción. La reproducción biológica, la reproducción de la autoconciencia y la conducta, la reproducción política y la reproducción societal. Todos estos niveles están conectados, aunque hayan sufrido de la "recaída en la inmediatez", es decir hayan borrado su propia génesis. Su origen está marcado por el concepto de epigénesis histórica, que significa que una estructura se forma a partir de resultados anteriores, es decir de estructuras previas (Samaja y Galende, 2009). Hay lazos o vínculos entre los estratos anteriores y los posteriores que si bien pueden cambiar de función o incluso de forma, permanecen como testigos mudos de una evolución que trabaja sobre materiales dados. Lo nuevo no es más que la recreación de los elementos que anteceden.

Lo que predomina es siempre una producción (una generación de lo nuevo) y una reproducción (un mantenimiento de lo dado). Hay una clara intención dialéctica en su formulación, pero actualizada con conceptos surgidos de las vanguardias científicas del siglo XX (teorías sistémicas, del caos y la complejidad, del constructivismo de Piaget, etc.). Lo que se busca es dar una descripción general que permita, por un lado, ubicar estratos con fines analíticos, por el otro, brindar una dinámica que posibilite observar el paso de uno a otro estrato.

En términos esquemáticos, Samaja nos introduce en tres diferentes niveles: el de los procesos fisicoquímicos, el de los fenómenos bióticos y el del fenómeno humano. Cada uno de estos niveles antecede al anterior (en un sentido lógico, pero también histórico). Al fin y al cabo como seres humanos no podemos dejar de tomar nota de los procesos físico-químicos 
que ocurren en nuestro cuerpo, así como tampoco podemos dejar de pensar en que somos sistemas bióticos (incluso se habla que somos ecosistemas, ya que tenemos más bacterias y microorganismos en nuestro cuerpo que células) y mucho menos obviar que como humanos somos el fenómeno donde el universo se hace autoconsciente, según la célebre expresión de Engels ( 2017).

En el primer nivel, el físico-químico, encontramos autoorganización, la materia se autoorganiza como en las "celdas de Bénard", estructuras disipativas las llama Prigogine (1997). Este fenómeno se produce cuando en un líquido sometido a una fuente de calor, las moléculas se "organizan" de tal forma, que optimizan la disipación de la incandescencia. En términos probabilísticos los chances de que se forme semejante estructura son bajísimos, sin embargo, la materia inerte, en condiciones alejadas del equilibrio, logra esa estabilidad estructural. La clave de esta forma de la "complejidad" radica en la dependencia, casi exclusiva, del medio externo. El medio en que se realiza es el de la materia, fundamentalmente en términos químicos.

En el segundo nivel, el de fenómenos bióticos, la autoorganización del nivel inferior (entendiendo siempre la jerarquía en términos puramente analíticos y nunca axiológicos) y la dependencia del medio externo se suman a las posibilidades de producción y reproducción. El segundo punto es muy claro, si algo caracteriza a los seres vivos es su capacidad de replicación. El primer punto es el que posibilita la evolución biológica. Como dice Samaja, este estadio se caracteriza por autonomizarse, hasta cierto punto, del medio ambiente. Si bien existe una dependencia, el mantenimiento de las estructuras puede prescindir, al menos por un tiempo, de las condiciones externas. El medio principal por el que se producen las nuevas formas, es el genético, sometido claro a la selección natural, tal como lo postulara Darwin (2012).

El último nivel, el del fenómeno humano, suma a la autoorganización de la materia y a la capacidad de reproducción y producción, la posibilidad de la representación, tanto individual como colectiva. Podríamos decir que aquí claramente emerge la cultura, entendida como toda aquella información no transmitida por medios genéticos y que encuentra en la imitación y el lenguaje, sus medios principales de transmisión (Diaz Córdova, 2011). Ese lenguaje natural, da paso a la escritura (más allá de las causas opresivas de su origen como fundamento de un estado prístino y estratificado) y esta a su vez a lo que Samaja llama "la producción de neo-objetos" donde interviene, claramente, el entorno computacional, aunque no es privativo de él y de hecho lo antecede. El medio principal es aquí el lenguaje. 
A su vez utiliza el mismo tipo de procedimiento para describir las características de lo que llama "reproducción humana". Su planteo está basado, como señalamos, en dos conceptos principales, producción y reproducción. Son ellos quienes proporcionan las bases para el cambio, pero también para el mantenimiento. Corsi et ricorsi (como repetía siempre en clase).

El primer nivel es el denominado "reproducción orgánica y biocomunal". Lo que se plantea aquí es que toda sociedad necesita reproducirse en sus niveles más básicos. Es necesario respirar, alimentarse, descansar, etc., de forma tal que, si no se cumplen esos procesos, el individuo no prospera, no supervive. Pero esta reproducción básica no es puramente individual, es también comunal, abarca la reproducción de las redes que constituyen el contexto.

El segundo nivel es el denominado "reproducción comunal-cultural". Aquí se postula que lo que se busca es la extensión del grupo íntimo, aparece la familia ampliada y las alianzas. El lenguaje ocupa un lugar central, ya que es preciso dominar el sistema simbólico. Nos dice Samaja que, en este nivel, no alcanza con la reproducción orgánica y biocomunal, sino que es preciso reproducir los sistemas simbólicos. Podemos decir que en este estrato emerge la "identidad", que es claramente societal y excede al individuo.

El tercer nivel es el denominado "reproducción estatal o política". Este estrato supera al anterior en tanto establece una comunidad basada en el territorio. Hay una supraorganización que regula y que en general se vincula con la aparición del Estado. Es decir, la reproducción del todo se autonomiza de la comunidad local y aún del vínculo íntimo (propio del primer nivel mencionado). Si existe el Estado, serán sus organismos e instituciones las que marquen el rumbo de la reproducción (y también de la producción).

El cuarto nivel es el denominado "reproducción societal". Este último estrato es el que se basa en la transformación necesaria de los objetos de la naturaleza, en "bienes" o "mercancías" (según la terminología marxista). Pero no solo es la producción de esos "bienes" necesarios (o superfluos) sino y fundamentalmente la circulación de estos. El "modo de producción" (en este caso capitalista) se impone y dirige la propia generación de un Estado, cuyo fundamento es una forma particular de propiedad privada.

Debemos aclarar que esta postulación no es de ningún modo una vuelta a un evolucionismo unilineal decimonónico. Ni que se plantee como 
un esquema de desarrollo, en un sentido eurocéntrico. Es simplemente un modelo para el análisis, donde se ponen de manifiesto cómo las relaciones del nivel inferior son suprimidas, conservadas y superadas por el nivel superior. Pero es claro que en el nivel empírico y de la acción, todas ellas actúan al mismo tiempo. Esta división, insistimos, es puramente analítica.

La idea de proponer esta suerte de esquema dinámico, tiene que ver con su planteo, en algún sentido holístico; es decir de no interpretar a las causas, en el proceso de salud-enfermedad-atención como elementos aislados. Citemos una frase del profesor Samaja que ilustra muy bien su idea:

El rasgo más destacado del paso de las configuraciones biológicas a las configuraciones culturales consiste en que las formaciones culturales no están fijadas sólo en el soma, como sucede con las especies biológicas, sino, también, y de manera crecientemente dominante, en el campo de lo imaginario, primero, y en las producciones simbólicas, después. (Samaja, 2009, p. 86)

Es decir, no se puede comprender lo que sucede en la sociedad, sin tomar en cuenta el contexto de los niveles orgánico, comunal y estatal, como antecedentes lógicos pero que temporalmente actúan al mismo tiempo. Esta mención al contexto no es simplemente la enunciación de una suerte de escenografía sobre la que se monta la comedia humana. Antes bien es parte integrante de las prácticas y representaciones tanto de los individuos como de las sociedades. La recaída en la inmediatez es la que posibilita que sus influencias queden oscurecidas o que se postulen como mero telón de fondo. Pero el planteo samajiano implica que el efecto de estos niveles se siente y afecta en todo momento.

Si bien el trabajo de Samaja está orientado fundamentalmente al proceso de salud-enfermedad-atención y a brindarles herramientas epistemológicas y metodológicas a los profesionales de la salud; lo cierto es que su modelo nos permite intentar comprender cómo interaccionan y se autoinfluencian lo que aquí denominamos ciclos de vida biológicos y ciclos culturales.

Básicamente el libro que tomamos como base, "Epistemología de la Salud", es un gran argumento en contra de los reduccionismos. Incluso nos atrevemos a señalar que toda la obra del profesor Juan Samaja es una gran lucha contra los reduccionismos de todo tipo. Tanto los biológicos 
como los culturales y dentro de cada una de esas esferas con los reduccionismos internos que siempre aparecen. La clave es intentar abordar la problemática en su complejidad manifiesta.

Debemos pues aclarar qué entendemos por reduccionismo, ya que pensamos que no puede ser usado como explicación monocausal, aunque reconocemos la importancia que puede tener en determinado momento del proceso de investigación.

En general se suele hablar de reduccionismos cuando explicamos un fenómeno por una sola causa. Por ejemplo, la sociobiología intenta explicar la conducta humana (sea cual fuere) como un efecto del condicionamiento genético. Llegando al absurdo de plantear que actitudes que no existían durante el proceso de hominización y por lo tanto de consolidación más fuerte de nuestra dotación genética, como por ejemplo fumar, están condicionadas por nuestro ADN. Determinar que la causa de la tuberculosis es el Bacilo de Koch, sin atender al resto de las condiciones que posibilitan la enfermedad, es incurrir en un reduccionismo (aunque, admitámoslo, más poderoso que el del ejemplo anterior). Pero que se postulen otras causas, como las condiciones de vida, la predisposición, etc., no quiere decir que no se reconozca al agente etiológico; es simplemente plantear que la complejidad de la enfermedad implica asumir todas las causas identificadas, proponiendo en todo caso, pesos distintivos a esas razones.

\section{Ciclos de vida biológicos}

Si bien en la introducción mencionamos que las etapas del ciclo de vida biológico son equivalentes para todos los seres humanos, seríamos necios si no ponderáramos el contexto en el que estas etapas toman lugar. La aparición de los eventos es universal, pero no lo es su ocurrencia y en algunos casos ni siquiera su semiología básica. Claro que partimos de la idea (y del hecho no refutado y cada vez con mayores evidencias) de que la humanidad, en cuanto a su origen es una sola, aparecida en África, una especie de primate, que es la única especie viva del género Homo (si bien cada vez es más claro que poseemos genes de Neandertales y de Homo denisova) (Roberts, 2010; Warren, 2018). Y que las fuerzas que guían a la evolución de la vida en la tierra son las que señalara Darwin hace ya más de 150 años. Azar y selección natural, es decir creación de variabilidad por diferentes mecanismos y un medio ambiente que selecciona, en función de sus propias características momentáneas, aquellos que pasarán la prueba(Darwin, 2012; Gould, 2002). 
En este caso, lo que nos importa es que, por un lado, tenemos todos los Homo sapiens los mismos eventos en el ciclo de vida; por el otro es que el medio ambiente (y necesariamente debemos contemplar a la cultura como parte de este), pueden modificarlos, sea acelerándolos o bien haciéndolos desaparecer. Pongamos un ejemplo claro antes de entrar en tema. La menarca (primera menstruación) claramente es un fenómeno que ocurre de forma universal en las hembras de la especie humana (y obviamente también de otros mamíferos, aunque se denomine ciclo estral y no menstrual); sin embargo, hay factores ambientales que pueden acelerarla, retrasarla o incluso suprimirla. Desde patologías hasta problemas alimentarios (sea por restricciones o abusos), toda la gama puede expresarse y así modificar y particularizar lo que, a priori, podría parecer un evento universal.

Barry Bogin, en su libro Patterns of human growth, nos plantea dos cuestiones importantes desde un punto de vista teórico, que permiten comprender mejor la problemática. Por un lado, dice que el orden de los eventos que él propone es arbitrario, de hecho dice literalmente que es uno de los órdenes posibles. Arbitrario, entendemos nosotros, en el sentido que daba Samaja a la construcción de las categorías, es decir como algo que nos brinda absoluta libertad, pero a la vez se ve restringido por los objetivos y el marco teórico en el que nos movemos. "La realidad puede ser descompuesta en infinitas variables", sentenciaba en sus clases el profesor Samaja, dando a entender que, a priori, no hay nada en el mundo que determine de qué manera se deben agrupar las cosas. Por otro lado, Bogin plantea que en sentido estricto el curso de la vida es cíclico y que una cosa es lo que sucede individualmente y otra cosa lo que acontece poblacionalmente (Bogin, 1999). Aquí también el planteo epistemológico es cauteloso y superador, cauteloso en tanto no cae en la "falacia de la concretidad mal aplicada" (es decir, no confunde el modelo con la realidad) y superador porque propone un esquema con fuerza heurística.

Los ciclos biológicos que propone Bogin son: a) la vida prenatal y los tres trimestres del embarazo; b) nacimiento y el período posnatal; c) infancia, desde el segundo mes de vida hasta el destete completo (generalmente a los 36 meses, el enfoque de Bogin es multicultural, tomando ejemplos de diversas culturas no exclusivamente la nuestra); d) niñez, desde el tercer año hasta el séptimo año de vida; e) juveniles, desde los 7 años hasta los 10, para las niñas y hasta los 12 para los niños; f) pubertad, es un evento muy corto en términos temporales; g) adolescencia, tiene una duración de entre cinco y ocho años luego de la pubertad; h) adultez, desde los 20 años hasta el final de la edad reproductiva; i) senectud, desde el final de la edad de procreación hasta la muerte (Bogin, 1999). 
Debemos aclarar que el profesor Bogin cuando plantea su esquema de los ciclos de vida, está tomando en cuenta siempre la problemática social. De hecho, el libro trata sobre los patrones de crecimiento en función de la inequidad social. Pero a los efectos de nuestro propio artículo, vamos a forzar una lectura con un anclaje estrictamente biológico, simplemente para ilustrar nuestro punto de vista. Solicitamos al lector que no juzgue de ningún modo como reduccionista el excelente trabajo del profesor Barry Bogin. Es el sentido general del presente texto el que nos obliga a tener una visión forzada que es la que aquí exponemos.

Desarrollemos un poco, en función del planteo que hace Barry Bogin, cada una de estas etapas. El autor nos aclara que la duración de cada uno de los estadíos está basada en datos tipo la media, la mediana o el modo, es decir son medidas de tendencia central, que no necesariamente indican un rango de variación normal. También nos comenta que este proceso de desarrollo es continuo y que la separación en etapas es puramente artificial, con el objetivo simple de comprender mejor todo el proceso.

La vida prenatal es un complejo desarrollo de una multiplicidad de elementos biológicos que dan forma al Homo sapiens. Durante las primeras semanas después de la concepción, todos los mamíferos nos parecemos; a partir de la octava semana los rasgos fenotípicos son claramente humanos. Los dos principales procesos principales que se aplican en este período son la hiperplasia y la hipertrofia. El primero responde a la división celular, a la mitosis, mediante la cual se crean células nuevas. El segundo responde al crecimiento en tamaño de cada célula individual. En definitiva, todo el desarrollo es cambiante, el cuerpo humano a lo largo de toda su vida está en un "permanente estado de descomposición y reorganización” (Bogin, 1999).

El nacimiento es la interfaz entre la vida dentro del útero y la vida fuera de él. Se pasa de un ambiente donde todo está dado por el cuerpo de la madre, a un ambiente donde todo depende del propio cuerpo. De un ambiente líquido a un ambiente rodeado de aire. Es uno de los momentos más complicados de toda la vida, las tasas de mortalidad en el nacimiento y el período neonatal (el primer mes de vida) siempre son más altas que en otros períodos antes de la adultez (Bogin, 1999). Uno de los principales problemas en esta etapa de la vida es el bajo peso al nacer, que puede condicionar tanto la vida como la muerte, así como afectar el resto del desarrollo del individuo. Durante este período, en general y a lo largo de la historia de la especie humana, el principal alimento siempre es la leche materna. 
La infancia, hasta los tres años, se caracteriza por la velocidad del crecimiento. La alimentación suele ser por vía de la lactancia materna, sobre todo debido a que aún los individuos no poseen dientes. Si bien en nuestro medio (urbano) se plantea que la lactancia es obligatoria hasta los seis meses, la OMS plantea un rango que va desde los seis meses (al menos) hasta los dos años de vida (OMS | Lactancia materna, s.f). En poblaciones de cazadores recolectores el destete puede ocurrir tan tarde como a los tres años (Bogin, 1999). Claro que no se trata de lactancia exclusiva, sino que se van incorporando otro tipo de alimentos. Durante esta etapa se observa claramente cómo el sujeto va adquiriendo capacidades cognitivas notables. Arranca sin poder levantar siquiera la cabeza (al mes de vida), hasta hablar, caminar y hacer algunas tareas de precisión (a los tres años).

La niñez, que va desde los tres a los siete años, es un período que se caracteriza porque el crecimiento físico se hace mucho más lento, en relación con el período anterior. Al principio de este momento, los niños requieren de una alimentación particular, que tome en cuenta el pequeño tamaño de sus dientes y también de sus estómagos, es la época de las papillas. En general, hacia el final de la etapa, ya se adquieren los hábitos alimentarios de los adultos (salvo excepciones, al menos en nuestra sociedad, como el café, el alcohol o los picantes). Hacia los seis años aparecen los primeros molares que coinciden con que el cerebro alcanza su peso máximo. Esta es una característica primate, si bien en los sapiens se da en forma un poco más tardía.

El período de los individuos juveniles dura relativamente poco tiempo, entre los siete y los diez años. Puede ser definido como la época en que los prepuberales ya no dependen de sus madres para la supervivencia (Bogin, 1999). Esta definición proviene del estudio de los mamíferos en general, pero es válida para los sapiens. Para este momento de la vida, los individuos ya adquirieron una serie de habilidades motoras, que les permiten ser cada vez menos dependientes de sus cuidadores. El ritmo de crecimiento es el menor desde el nacimiento. Es en esta etapa donde comienza a marcarse el dimorfismo sexual, ya que, en general, los individuos femeninos terminan esta etapa unos dos años antes en promedio, lo que refleja el adelantamiento de la adolescencia entre los sujetos femeninos.

El arranque de la adolescencia está caracterizado por una aceleración del proceso de crecimiento y desarrollo. En esta etapa comienza la maduración sexual y social, cuyos caracteres fenotípicos son fácilmente observables (aparición del vello púbico, cambio en la tonalidad de la voz, etc.). A su vez es aquí cuando los individuos empiezan a adquirir los 
patrones de conducta de los adultos de su especie. En la adolescencia ocurre un crecimiento acelerado de todos los tejidos del cuerpo. Es el famoso estirón adolescente; a diferencia de otros primates, en el Homo sapiens este proceso es mucho más intenso. Otra diferencia con otros grupos de primates es la duración, mucho mayor en los humanos, para alcanzar la madurez sexual.

Una de las marcas de la adultez es la detención del proceso de crecimiento de la talla. Esto comienza entre los 18 y los 21 años. Otro de los hechos que determina la llegada a la adultez es que se alcanza la madurez reproductiva. Es interesante como Bogin distingue fertilidad (que se alcanza durante la adolescencia) de la madurez reproductiva (que es la capacidad de llevar un embarazo a buen término y poder criar a sus hijos de tal forma que puedan sobrevivir). Muchos de los tejidos del cuerpo pierden la capacidad de la hiperplasia (la división celular), pero muchos pueden seguir creciendo por hipertrofia (el crecimiento de cada célula). Esta etapa se caracteriza por cierta estabilidad en el crecimiento y por ser el momento en que los individuos son más resistentes a enfermedades infecciosas y otro tipo de patologías.

La senectud o envejecimiento es el período cuando se manifiesta un descenso en la habilidad para adaptarse a los problemas que provienen del medioambiente. Uno de sus hechos biológicos más notables es el de la menopausia, que se da únicamente entre los individuos de sexo femenino. A nivel celular hay un declive en la capacidad de ciertas células que no pueden replicarse (hiperplasia) para poder regenerarse, con lo que su funcionamiento queda evidentemente resentido. No hay explicaciones claras acerca de porqué sucede este proceso de declive. Si bien hay diferentes hipótesis, una de ellas es la plieotrópica que plantea que genes que pudieron ser beneficiosos durante el proceso de crecimiento, se tornan deletéreos en esta etapa; lo cierto es que no hay una teoría que tenga consenso en la comunidad científica (Bogin, 1999).

Hemos presentado muy sucintamente los estadíos de los ciclos de vida desde una perspectiva exclusivamente biológica, es decir desde una postura abstracta y analítica. Hemos manipulado los conceptos del profesor Bogin, simplemente a efectos expositivos, en función de la necesidad de plantear posturas contrastantes, para proponer luego nuestra propuesta basada en el pensamiento complejo.

Vamos ahora a presentar los ciclos de vida desde una perspectiva cultural. También aquí manipularemos la información, concentrándonos 
únicamente en los aspectos culturales de estos ciclos, eliminando los fundamentos biológicos con los que claramente se interrelacionan.

\section{Ciclos de vida culturales}

Es difícil encontrar literatura que plantee los ciclos de vida culturales que son vividos por los individuos en una forma general y enfatizando los puntos en común. Esto en principio porque dada la enorme variabilidad que existe entre las diferentes culturas, hace difícil encontrar patrones universales. Pero además porque la literatura antropológica está ciertamente impregnada de un particularismo que, muchas veces, se aplica de manera no crítica y funciona como axioma o supuesto. Lo cierto es que el atributo de particular y universal están imbricados. No puede existir uno sin el otro, desde el momento en que son atributos claramente relacionales. En general, que se diga de algo que es particular o que es universal, depende más de las características seleccionadas que de algo ontológico.

El planteo particularista tiene un sentido importante en términos políticos, ya que implica necesariamente que no hay una cultura mejor que otra. Pero llevado al extremo puede ser peligroso, justamente en términos también políticos. La justificación, en nombre de la "cultura", de cualquier atrocidad no debe ser admitida. El problema es complicado y tal vez las únicas herramientas para no caer ni en el exceso ni el defecto sean la reflexión permanente y el análisis a conciencia. Lo mismo sucede cuando se plantean aspectos universalizables; por un lado, tiene un criterio positivo, de hermandad de toda la humanidad, de la unidad psíquica de la especie; por el otro lado puede ser peligroso en términos políticos: imponerle condiciones a una cultura en el nombre de una supuesta "universalidad".

La estrategia que nos planteamos en este acápite es la de poder hacer una abstracción de aspectos culturales vinculados con los diferentes ciclos que atraviesan los individuos a lo largo de su vida. Ahora bien, encontrar literatura sobre todas las culturas existentes es una tarea prácticamente imposible. Mucho más si consideramos a culturas que existieron a lo largo de la historia. Esto hace que nuestra "muestra" no sea ni mucho menos completa. Su incompletitud es parte de la imposibilidad concreta de obtener la información adecuada.

Una fuente que usamos es la base de datos que comenzó a recolectar George Murdock allá en la década de los 30 y que fuera conformando a lo largo del tiempo el llamado Human Relation Area File (Murdock, 1967). La idea de este repositorio es la de incorporar rasgos de las diferentes 
culturas bajo un esquema universal, en donde sea sencillo ubicar los distintos aspectos de interés en todas las culturas. Por ejemplo, si uno busca matrimonio, aparecen todas las formas recolectadas de uniones y alianzas en múltiples culturas. Es cierto que se pueden criticar las categorías utilizadas; ¿es exactamente un matrimonio lo que practican en la cultura tal? Claro, la respuesta depende del tipo de definición que se postule. En nuestro caso, la universalidad es simplemente un punto de partida que de ningún modo pretende eliminar la riqueza subyacente de la propia variabilidad cultural.

Otra fuente posible son las diferentes etnografías que hemos leído a lo largo de nuestra carrera como antropólogos, pero de las que no tenemos un registro formalizado, sino que se encuentran sus contenidos, dispersos por la memoria y por tanto en la bibliografía consultada. No es nuestro interés establecer un documento canónico sobre la universalidad de ciertas prácticas o la particularidad en su implementación. Nuestro objetivo es armar y ofrecer un esquema que luego nos permita utilizarlo para dar cuenta de cómo pueden ser interpretados los dos niveles planteados, el cultural y el biológico. Es en definitiva una excusa para aplicar el modelo de pensamiento complejo que emerge de los trabajos del profesor Samaja y que hunde sus raíces tanto en las ideas de Gregory Bateson y su discusión sobre los tipos lógicos, como en ciertos conceptos de Hegel.

Los tipos lógicos son un argumento explicativo que utiliza Gregory Bateson para dar cuenta de diferentes tipos de fenómeno, desde el aprendizaje hasta la comunicación (Bateson, 1980). La idea original proviene de la teoría de conjuntos desarrollada por Bertrand Russell y Alfred Whitehead y consiste básicamente en crear categorías inclusivas, es decir que pueden estar unas dentro de otras. Estos diferentes niveles de categorías, cada uno de los "tipos lógicos" suelen tener aspectos inconmensurables entre sí, en el sentido que cuando una señal no se interpreta dentro del tipo lógico adecuado, suele suceder un problema de comunicación, que puede provocar una pelea o un chiste, si se trata de comunicación oral (Bateson, 1980). Cada tipo lógico sirve de contexto al nivel más bajo y si bien en los sistemas hay comunicación entre cada uno de ellos, la interpretación errónea, la confusión de tipos lógicos, suele generar inconvenientes comunicacionales.

Una cosa es claramente segura, la cultura no permanece indiferente ante los acontecimientos que jalonan la vida de los individuos. Ya desde Durkheim se habla de hechos sociales, como aquellos hitos que atraviesan toda la vida de una sociedad, que afectan a todos los aspectos, 
económicos, políticos, etc. (Durkheim, 2005). La antropología plantea que no solo las normas sociales están reguladas por la cultura (lo que se denomina Eidos), sino que las emociones están determinadas culturalmente (lo que se denomina Ethos)(Bateson, 1998). No somos libres de elegir qué es lo que nos va a emocionar, qué es lo que nos enoja o qué es lo que nos alegra. Estamos regidos por la cultura y eso nos hace, mal que le pese a nuestro ego, que por cierto pesa mucho, unos seres bastante predecibles.

Si bien estos ciclos biológicos que presentamos no necesariamente cubren todas las manifestaciones culturales (la cultura también trabaja sobre fenómenos propiamente culturales), es cierto, a la inversa, que esos eventos biológicos tienen un correlato fuerte en la propia cultura. Vamos a tratar de poner, entonces, ejemplos que ilustren el punto; para luego intentar encontrar los puntos en común en función del marco teórico elegido. Dada la diversidad cultural y el hecho de que cada cultura define sus acciones y valores en función de problemáticas diferentes, no necesariamente todas las culturas tendrán respuestas a los mismos interrogantes. De allí que pongamos ejemplos de algunas pocas sociedades, incluyendo nuestra propia cultura. La idea es, entonces, desarrollar algún dispositivo conceptual que permita empezar a resolver el problema de los vínculos entre la naturaleza y la cultura, un problema que ya es un clásico en la antropología.

\section{Embarazo}

Comencemos entonces por el primer punto del ciclo de vida biológico. La vida prenatal y los tres trimestres del embarazo. En general, durante el embarazo y sobre todo si es un embarazo primerizo, el grupo de referencia de la madre, suele prestar una atención particular, repartiendo consejos y relatando experiencias vividas. En nuestra propia cultura, la respuesta (muy propia del sistema simbólico impuesto luego de la Segunda Guerra Mundial donde la atención en salud, propia del estado de bienestar, se masifica) está dada por las visitas al ginecólogo y los controles obstétricos. El babyshower es una costumbre que se da en muchas culturas, además de la occidental. Y en muchas culturas es el acto que indica que la cultura matriz está por recibir a un nuevo miembro. Por ejemplo, entre los Azande, la mujer debe observar ciertas prohibiciones alimentarias, que suelen ser similares a las que observó su madre cuando estaba embrazada de ella (Baxter y Butt, 1953). Entre los pigmeos Mbuti también se observan prohibiciones alimentarias (Turnbull, 1965). Entre los isleños de las Islas Marshall, la mujer durante el embarazo toma baños en las lagunas y se masajea el abdomen para fortalecer los 
músculos (Erdland y Neuse, 1914). Como puede observarse en estos ejemplos etnográficos de diferentes partes del mundo, la cultura establece pautas para poder encarar el problema biológico del embarazo.

\section{Nacimiento}

El nacimiento es la segunda etapa y aquí también la cultura tiene algo que decir. La ceremonia del ingreso formal del nuevo individuo no necesariamente se da el propio día del nacimiento. Sin ir más lejos el bautismo católico no se realiza en el mismo momento del parto, sino algunos meses después. Se nombran padrinos y se realiza una fiesta en la que participa la familia y los allegados. En algunas culturas se asocia a esta ceremonia con la adjudicación de un nombre. De algún modo marca la pertenencia a la sociedad humana del nuevo integrante. Es cierto que el bautismo católico no se aplica únicamente a niños sino a cualquiera que quiera integrar esa fe, sin importar su edad; pero lo cierto es que, entre familias practicantes de esa religión, la mayor parte de los bautismos se dan en los primeros meses de vida. Entre los pueblos de Labrador, ningún hombre puede estar presente durante el parto, muchas veces la placenta o bien se quema o bien se entierra (McGee, 1961). Los Yurok poseen un ritual para el nacimiento, mediante el cual se duplica el espíritu de la persona; mientras recita, toma cenizas del fuego y las frota sobre el abdomen de la mujer que está dando a luz (Keeling, 1992). Entre los Maoríes para aliviar los dolores del parto se calientan piedras que se cubren con hojas y arriba de ellas se coloca una manta, de este modo le dan a la embarazada un baño de vapor (Buck, 1952).

\section{Infancia}

El período que biológicamente se denomina infancia y que abarca los tres primeros años de vida, no tiene una ceremonia exclusiva, tal como se observa en la etapa anterior. En algunas culturas se realizan regalos y rituales al momento del destete (que es cuando termina esta etapa), ya que implica que se abandona la alimentación de exclusividad materna para empezar a incorporarse al mundo alimentario de los adultos. En nuestra propia cultura, si bien no hay una ceremonia específica, implica el paso a comer en la mesa como el resto de la familia. En general, en nuestra cultura, las madres no dan de amamantar a sus niños en la mesa donde se come. En estudios que realizamos (Aguirre et al., 2015) notamos que la gente en general no considera comida a la lactancia y no le da el lugar social que tiene la comida compartida de niños y adultos. Entre los Huicholes, la madre inventa una clase de lenguaje con el cual se comunican con sus hijos durante la infancia y si bien es ininteligible, lo cierto es que es ideal 
para comunicar emociones (Zingg, 1938). Entre los Mapuche, durante la infancia, empiezan a cuidar de los rebaños y a adquirir algunas responsabilidades de los adultos (Titiev, 1951). Entre los Kazak de Asia, los niños en esta etapa son entrenados por los padres y las niñas por las madres, en esta edad es cuando practican la circuncisión, que es acompañada por una fiesta organizada por el padre (Hudson, 1938).

\section{Niñez}

El período que denominamos niñez abarca, aproximadamente, desde los tres años hasta los siete años. En nuestra cultura es el momento en que se ingresa a la escuela primaria, fenómeno que tiene un impacto simbólico importante. Entre los Guayaki del Chaco Paraguayo, nos cuenta Pierre Clastres que los niños reciben un arco pequeño y las niñas un cesto pequeño (Roa Bastos, 2011). Estos elementos son centrales en la estructura de esa sociedad y en las relaciones entre los sujetos en su vida adulta. Claramente es el momento donde empieza con fuerza el proceso de socialización, donde se adquieren los gustos y también se empiezan a comprender aquellas situaciones que moldean las emociones. En nuestra cultura es el momento, por ejemplo, cuando los niños suelen adquirir la pasión por sus equipos de fútbol preferidos. Entre los pueblos de Ruanda, los niños Batutsi hacen deporte, bajo la supervisión del jefe Mwami, particularmente una danza denominada guhamiriza (Pagés y Scholl, 1933). Los niños Hadza son reconocidos por su productividad como recolectores, entre los siete y los 10 años ya son capaces de proveerse con la mitad de los requerimientos kilocalóricos diarios, trabajando aproximadamente dos horas (Crittenden y Blurton Jones, 2019). Si observamos a los pueblos rurales irlandeses veremos que luego de la primera comunión a los siete años, el niño empieza a interactuar más con sus hermanos mayores, al tiempo que comienza a cantar algunos coros con su padre y otros adultos de la aldea (Arensberg et al., 1940).

\section{Juventud}

Los individuos juveniles son aquellos que tienen entre los siete y los 10 años para las niñas y los 12 para los niños. Dentro de la religión católica es el momento en que los individuos suelen tomar la "comunión", es decir ingresan en el rito de manera completa, más allá de que el comienzo sea el bautismo. Es un momento de consolidación de ciertas prácticas culturales. En nuestra propia cultura ya aprenden a leer y a escribir y esto es aprovechado por las industrias para lanzar campañas publicitarias dirigidas a este sector. Nos guste o no, el capitalismo es la forma económica de nuestra sociedad y por lo tanto el marketing forma parte de las pautas culturales 
y claramente del proceso de socialización. Estas pautas publicitarias se basan en la capacidad de los individuos de esta edad de hacerse oír frente a los padres y mayores. Entre los Banyoro del África Central, la vestimenta es la que marca las edades, aunque no sean vestimentas propiamente dichas, sino collares y pulseras; a los seis años usan una piel de vaca, luego de la iniciación puede usar dos pieles, las chicas suelen usar un taparrabos y a veces una piel que las cubre (Roscoe \& Mackie Ethnological Expedition To Central Africa, 1919-1920, 1923). Entre los Kurdos es la época en que se aprenden los versos del Corán, los Kurdos no consideran a la niñez como una etapa en sí misma, sino como una preparación para la adultez, es el tiempo del aprendizaje (Hansen, 1961). En Nueva Guinea los individuos juveniles aprenden a blanquear los lados de las canoas con jugos hechos de semillas. Ellos saben tallar, pero no labrar; si bien pueden hacer antorchas de bambú para las expediciones nocturnas, no saben nada de carpintería, salvo de lo que recuerdan de su infancia, cuando pasaban más tiempo en compañía de sus padres (Mead, 1930).

\section{Pubertad}

La siguiente etapa es la pubertad. Como se plantea en el apartado de los ciclos biológicos, esta etapa dura muy poco, sin embargo, posee una importancia central, ya que está vinculada con el comienzo de la maduración sexual. Entre los pueblos pastores, por ejemplo, es el momento cuando los pequeños adquieren sus primeros animales. El ritual más famoso seguramente sea el de la religión judía, el Bar Mitzvah (para los niños) y el Bat Mitzvah (para las niñas). De algún modo este es el período en que aparecen algunas circunstancias que tendrán luego una importancia grande en la vida adulta. Básicamente la aparición de los caracteres sexuales y la adquisición de ciertos rasgos propios del adulto. En algunas culturas es cuando se realizan los rituales de iniciación. En las islas caribeñas este período es tratado como un tiempo cuando se realizan las escarificaciones y en donde las mujeres obtienen su máscara (Gullick, 1976). Entre los Innus de Labrador se practicaba una suerte de reclusión así como un conjunto de prohibiciones alimentarias para con los púberes (Burgesse, 1944). Los Bosquimanos San tienen una serie de complicados rituales tanto para mujeres como para hombres, los pasos que se siguen en los rituales son una suerte de pruebas en función de sus futuras habilidades como adultos (Fourie, 1928).

\section{Adolescencia}

Llegamos a la adolescencia. Si bien este período nos parece muy obvio, lo cierto es que, en Occidente hasta después de la Segunda Guerra 
Mundial, prácticamente no existía. Había una suerte de salto de la pubertad hacia la adultez, seguramente motivado por los patrones socioeconómicos que moldeaban a la cultura. Cuando el capitalismo descubre que la adolescencia puede ser un mercado muy importante, empieza a diferenciarse desde un punto de vista cultural. La independencia de los individuos en este período, al mismo tiempo que su capacidad para influir sobre los mayores que aún los tienen a cargo, los convierten en un mercado muy apetitoso para las industrias y empresas. La adolescencia es el período cuando el entrenamiento para la vida adulta es el más fuerte. Es una etapa llena de rituales no formales en nuestra sociedad, por ejemplo, las primeras borracheras o los primeros encuentros sexuales. Entre los Nyakyusa, del África, cuando los individuos llegan a la adolescencia forman su propia aldea, ya que consideran que las actividades sexuales de las generaciones diferentes deben mantenerse separadas y que los mejores vecinos son los de la misma generación (Kenny, 2011). Para los Kwoma de Nueva Guinea, durante la adolescencia van adquiriendo cada vez más responsabilidades en el trabajo de manejar los cultivos; además de empezar a estrechar lazos con los adultos de su familia que forman parte de los grupos de cultivo, de esa manera se comienza a ser adulto (Whiting, 1941). En América del Sur, los Warao cuando llegan a la adolescencia, adoptan las características identitarias del héroe cultural Haburi, el inventor de las canoas; vagabundean de casa en casa y de aldea en aldea, en algún momento una adolescente comienza a seguirlo y con la excusa de ir a cazar pájaros, abandonan la aldea durante semanas (Wilbert, 1976).

\section{Adultez}

La adultez es la siguiente etapa definida en el apartado anterior. En algún sentido podemos decir que uno de los rituales que se practican en esta etapa es el del matrimonio, tanto en términos religiosos como civiles. En toda sociedad, las ceremonias que se practican tienen consecuencias sobre la vida de los individuos. Es por ello que en una sociedad como la nuestra, el matrimonio implica un contrato social, de allí la enorme importancia que tiene la aprobación del matrimonio igualitario, que claramente equipara derechos. En otras culturas o en Occidente en otras épocas, el matrimonio se practicaba (o se pactaba) a edades más tempranas. Sin embargo, dado el exagerado lugar que ocupa el individuo en el capitalismo, es necesario otorgarle, en los primeros momentos de este período, la mayoría de edad y por lo tanto el otorgamiento de los derechos plenos y la demanda de las responsabilidades y obligaciones concretas. Para los Yeranias, de Asia Menor, el matrimonio implica el paso hacia la adultez, sin embargo toda la preparación para el casamiento es responsabilidad de los padres (Hirschon, 1989). 
En Japón, el grupo Ainu, como casi todas las sociedades de cazadores recolectores tienen muchas reglas culturales que deben respetarse; en el caso del paso a la adultez, los Ainu adolescentes (que ya poseen una importancia económica central) deben aprender el lenguaje de los adultos plenos, así como el lenguaje religioso y las formas que adoptan los rituales (Ohnuki-Tierney, 1974). Los habitantes del Atolón Ulithi, en Oceanía, definían el pasaje a la adultez como una serie de aprendizajes vinculados con la religión y la magia, con la genealogía y el uso de la tierra; para ingresar en la adultez plena los Ulithi consideraban una serie de cambios físicos, como la pérdida del tono muscular, la aparición de canas en el cabello y la pérdida de algunos dientes (Lessa, 1950).

\section{Senectud}

La senectud es la última etapa de los ciclos biológicos que hemos definido. Claramente para nuestra cultura, al ser un período de inactividad y retiro (la jubilación), este grupo etario está prácticamente fuera de las consideraciones. O mejor dicho se lo considera improductivo y por lo tanto no hay un tratamiento particular. Si bien desde el Estado se implementan algunas medidas para que no caigan en el abandono completo o dejados a la suerte de sus familiares, lo cierto es que los gobiernos de corte conservador siempre están dispuestos a quitarles algo. Más allá de la cuestión política, desde el punto de vista cultural, esta etapa suele tener una importancia muy grande para los más chicos. En contextos etnográficos son los vínculos entre los ancianos y los niños (mediados por el cuidado, entre otras actividades) los que mantienen viva la historia de la propia cultura. En Bali, todos los adultos mayores son denominados como "abuelos", como una señal de respeto; entre las mujeres, al llegar a la edad adulta, ellas suelen actuar como sacerdotisas y son esenciales en los festivales religiosos (Covarrubias, 1938). Entre los Chorote, de Sudamérica, los ancianos son los que están autorizados a comer cuervos y búhos debido a su conocimiento acerca de los espíritus malignos, no a cualquier edad se puede comer cualquier animal (Rosen, 1924). Entre los Inuit del Ártico, los adultos mayores siguen siendo productivos, participando de las actividades de caza junto a sus nietos; las mujeres siguen también cociendo, generando recursos; todo hasta que sus fuerzas los abandonan y quedan confinados en los campamentos (Condon, 1987).

Vamos ahora, en el siguiente acápite, a intentar de establecer los vínculos entre un nivel y el otro, tratando, desde una perspectiva basada en las teorías de la complejidad, de encontrar un operador que nos permita pasar, en forma coherente, de uno a otro tipo lógico. 


\section{Una síntesis en sentido estricto}

Un elemento central para tomar en cuenta es que, así como la biología moldea la cultura, la cultura puede a su vez modificar la biología. Por ejemplo, la fisiología del aparato digestivo del Homo sapiens exige que la cultura desarrolle todo un conjunto de dispositivos (siendo la cocción el más saliente) para poder dar cuenta de esas necesidades (Ungar, 2006). A la inversa una cesárea implica una modificación de la acción biológica por intermedio de una pauta cultural. Este tipo de interacciones se da en prácticamente todo momento. Y si se establece una distinción en función de un antecedente y un consecuente es simplemente a efectos expositivos. Porque si bien hay una biología que antecede, en términos temporales, al menos durante el proceso evolutivo de las especies, lo cierto es que ya el Homo sapiens aparece en la Tierra dotado de unas cuantas herramientas culturales. Esto se observa cuando se analizan los restos de homínidos anteriores que contaban con instrumentos y tecnologías diversas (Roberts, 2010).

Podemos decir, en términos cibernéticos, que se da una interacción mutua entre uno y otro ámbito. Un pequeño cambio en un elemento de uno de ellos, puede desencadenar un cambio inmenso en el otro. No se trata de encontrar un orden causal, del tipo "huevo o gallina", sino de interpretar la simultaneidad de una forma tal que permita comprender todo el proceso. Y para ello nos valemos de los conceptos expresados por el profesor Samaja, acerca de la cuestión del proceso de salud-enfermedad. Y también tomaremos algunas ideas del profesor Bateson, ya que ambos, a pesar de las distancias temporales y geográficas comparten una misma forma de pensar.

Una idea central es la que postula Bateson, acerca de que en la estructura se encuentra inscripta la historia de ese objeto. No pueden borrarse las huellas de su génesis y si se sabe mirar, es posible reconstruir toda la trayectoria. La cultura no es un accesorio de la biología, sino que es la interfaz que la humanidad encontró para dar cuenta de los problemas que la naturaleza plantea. Pero eso no implica que la cultura siempre encuentre las mejores soluciones o que las soluciones encontradas no tengan efectos en el largo plazo. Un caso muy claro es el que sucede con la alimentación industrial, que generó por un lado la posibilidad de que mucha más gente acceda a los alimentos (debido a que se reducen los precios por la escala industrial de producción), pero a la vez genera los nuevos problemas de sobrepeso y obesidad (Aguirre, 2017). 
Otra idea notable que podemos utilizar es la de la recaída en la inmediatez, que se entronca con la expuesta en el párrafo anterior. La recaída en la inmediatez implica que, en el momento presente, las condiciones que le permitieron aparecer, se muestran oscurecidas, reducidas a una mínima expresión, que hace dificultosa la recreación de la trayectoria. Sin una mirada atenta y profunda, a conciencia, aquellas condiciones iniciales aparecen veladas, restringidas a pequeños espacios. Por ejemplo, el sistema medieval de los siervos de la gleba, con todas las limitaciones a su libertad, fue superado en el capitalismo por el sistema de libertad de trabajo (aunque sólo sea formalmente). Sin embargo, quedan espacios en el mundo (y aquí mismo en la Argentina) donde aún subsisten, con algunos parámetros cambiados, es cierto, pero que mantienen la estructura férrea de trabajar para pagar la deuda (el impuesto en la edad media) sin cobrar dinero y teniendo restringida la posibilidad de irse a otra parte (el anclaje a la tierra). La recaída en la inmediatez es la condición que hay que buscar con lupa para reconstruir, a partir de la estructura del fenómeno, su historia y su propia génesis.

También es importante tomar en cuenta las ideas de Bateson acerca de los tipos lógicos. Esta idea del antropólogo británico tiene su origen en la teoría de conjuntos de Bertrand Russell, quien planteaba que los elementos de un conjunto están en un nivel diferente que el propio conjunto. Esto implica que un elemento no puede ser el conjunto y el conjunto no puede ser su propio elemento. Hay una necesaria discontinuidad. Esto nos lleva a distinguir claramente los niveles y esta observación es necesariamente analítica. Debemos distinguir entre la realidad y los esquemas que utilizamos para describirla. Allí ya tenemos una primera distinción entre dos tipos lógicos. Ahora bien, en nuestras descripciones es posible que también encontremos la necesidad de dividir en tipos lógicos diferentes nuestros niveles de descripción (sin ir más lejos la distinción entre local y global responde a esta lógica). Postulamos entonces que entre los niveles biológicos y culturales existe una distinción de tipos lógicos; que son dos niveles que sólo existen a efectos del análisis y que en la realidad empírica se encuentran tan imbricados que, sin una reflexión, se hace imposible distinguirlos. Habría señales en los niveles inferiores que disparan cambios en los niveles superiores y a la inversa. Estas señales, un poco a la manera de Roy Rappaport en "Cerdos para los antepasados" funcionan como disparadores de cambios estructurales en los otros niveles (Rappaport, 2000). Como señales que son interpretadas por el nivel superior y donde se actúa en consecuencia. 
Supresión, conservación y superación parecen ser los otros conceptos clave para entender los vínculos dinámicos entre un nivel y otro dentro de la teoría de los tipos lógicos. Estas señales mencionadas que comunican los diferentes niveles, son en principio suprimidas, pero terminan siendo conservadas (esto proviene de un artilugio lingüístico que utilizó Hegel con respecto a la palabra Aufhebung, traducida al español como abrogar, pero que también significa preservar). Es decir, son oscurecidas o reducidas a su mínima expresión, lo que no implica que sean eliminadas. Samaja nos plantea que lo suprimido pierde su condición de autonomía, de pauta general. Lo conservado quiere decir que se conserva aún su capacidad de acción y aunque ya no tenga el poder para actuar en forma autónoma, mantiene su potencia para actuar, pero queda supeditada a la nueva estructura. La superación es la consolidación del proceso y la irrupción de la nueva estructura. Es la etapa final y, debido al carácter cíclico, el comienzo de un nuevo proceso de transformación.

Si bien en el esquema ideal el flujo va de un nivel hacia el otro en un sentido ascendente, lo cierto es que los fenómenos que se mapean contra ese esquema pueden obligar a modificar ese orden ideal. De hecho, el propio Samaja nos alerta que en fenómenos vinculados con los procesos de salud-enfermedad-atención, pueden darse situaciones en las que una señal se suprime, pero no se conserva y también pueden alterar su orden en cuanto al flujo de las señales. Esto es muy notable en cuanto como la cultura puede modificar situaciones estructurales básicas del nivel biológico.

Es probable que el lenguaje natural sea un impedimento para poder desarrollar este marco teórico y tengamos que empezar a pensar en términos algorítmicos para aplicar con rigor un pensamiento complejo. En definitiva, un algoritmo no es más que una regla o un conjunto de reglas que se aplican sobre un espacio dado, para resolver alguna clase de problema. Es decir que mediante la aplicación algorítmica se pueden observar en funcionamiento los momentos de supresión, conservación y superación, así como mantener en un orden claramente distinguible los niveles o tipos lógicos planteados.

Para cada una de las etapas definidas en el nivel biológico se pueden encontrar una o varias en el nivel cultural. Se puede verificar cómo la cultura suprime, conserva y supera esas señales enviadas desde el nivel biológico.

Pongamos por ejemplo el babyshower, se supone que es una presentación en sociedad del embarazo, sin embargo, al menos en nuestra 
sociedad capitalista, lo que pasa a un primer plano, no es la salud de la madre durante el embarazo, sino la cantidad de cosas que se le pueden regalar; hay una industria mucho más preocupada por las ventas que por lo que suceda con ese embarazo. La industria, en tanto pauta cultural, se monta sobre la señal biológica.

En el caso del bautismo católico se ve claramente como lo importante para la institución (la iglesia) es el alma, mucho más allá del fenómeno biológico del nacimiento. Lo que importa es que ese recién nacido pueda llegar al "cielo" en caso de muerte y no quede en el "limbo". Pero claro que sin nacimiento no puede haber bautismo; ambos niveles se necesitan, pero la cultura suprime, a la vez que conserva la señal biológica. Y a la vez la supera, ya que crea una estructura nueva (la religión del individuo), en donde el alma ocupa un lugar que antes no tenía (el antecedente es puramente lógico).

Para poder identificar cómo esos signos de la naturaleza son interpretados por la cultura que los envuelve es necesario observar cómo son resignificados y qué lugar ocupan en la nueva estructura. ¿Es la aparición de los caracteres sexuales lo importante del Bar Mitzvah? Evidentemente no, ya que el evento se dispara más por cronología, que por la biocronología. No importa si el individuo madura más tarde o maduró más temprano. Lo importante es que ya empieza a formar parte del mundo de los adultos.

El matrimonio, que en algunas culturas se desarrolla al final de la adolescencia, en otras en la adultez, en otras entre diferentes etapas del ciclo de vida (como era costumbre en Occidente, mujer adolescente, hombre maduro), no responde a una necesidad de reproducción. Si bien podemos decir que el disparador biológico marca el comienzo, aun cuando la mujer es adolescente puede ser fértil, aunque no haya alcanzado la madurez reproductiva, esa pauta es suprimida, conservada y superada y se transforma claramente en un acto vinculado con las alianzas sociales.

Hasta aquí hemos realizado una breve exploración e identificado algunos elementos que permitan ir construyendo un marco teórico para la comprensión del vínculo entre ambos niveles. Sospechamos que en una próxima etapa debe incluir algunos ejercicios algorítmicos, ya que es posible encontrar otro espacio de entendimiento cuando se manipulan, en una pieza de software, algunos conceptos teóricos. Es evidente que debemos hacer uso de ciertos atributos como las retroalimentaciones, la sensibilidad a las condiciones iniciales o la morfogénesis cuando se 
intenta indagar en la estructura de las relaciones entre la naturaleza y la cultura. Es probable que no hayamos alcanzado los objetivos propuestos, simplemente nos contentamos con haber dado los primeros pasos en esta búsqueda.

\section{Referencias}

Aguirre, P. (2017). Una historia social de la comida. Buenos Aires: Lugar Editorial.

Aguirre, P., Díaz Córdova, D. y Polischer, G. (2015). Comer y cocinar en Argentina hoy. Buenos Aires: FUNDASAP.

Aiello, L.C. y Wheeler, P. (1995). The Expensive-Tissue Hypothesis: The Brain and the Digestive System in Human and Primate Evolution. Current Anthropology, 36(2), 199221. Recuperado de https://doi.org/10.1086/204350

Arensberg, C.M., Conrad M., Kimball, S.T. y Warner, W.L. (1940). Family And Community in Ireland. Harvard University Press. Recuperado de https://ehrafworldcultures.yale. edu/document?id=er06-001

Ashby, W.R. (2015). Introduction to Cybernetics. Eastford: MARTINO FINE Books.

Aspray, W. (1993). John von Newmann y los orígenes de la computación moderna. Barcelona: Gedisa.

Bateson, G. (1980). Mind and nature: A necessary unity. London: Fontana.

Bateson, G. (1998). Pasos hacia una ecología de la mente. Buenos Aires: Lohlé-Lumen.

Baxter, P.T.W. y Butt, A. (1953). Azande, and Related Peoples of The Anglo-Egyptian Sudan And Belgian Congo. En Ethnographic Survey Of Africa, East Central Africa. International African Institute. Recuperado de https://ehrafworldcultures.yale.edu/ document?id=fo07-056

Bertalanffy, L.V. (2018). Teoría General de los Sistemas: Fundamentos, desarrollo, aplicaciones. México: Fondo de Cultura Económica.

Binford, L.R. (1991). En busca del pasado: Descifrando el registro arqueológico (2. ed). Barcelona: Ed. Crítica.

Bogin, B. (1999). Patterns of human growth (2nd ed.). Cambridge: Cambridge Press.

Buck, P.H. (1952). Coming of The Maori. Maori Purposes Fund Board; [distributed by] Whitcombe and Tombs. Recuperado de https://ehrafworldcultures.yale.edu/ document?id=oz04-003

Burgesse, J.A. (1944). Woman and The Child Among The Lac-St-Jean Montagnais. Primitive Man, 17(1 \& 2), 1-18.

Condon, R.G. (1987). Inuit Youth: Growth And Change In The Canadian Arctic. Rutger University Press. Recuperado de https://ehrafworldcultures.yale.edu/document?id=nd08-033

Covarrubias, M. (1938). Island Of Bali. Alfred A. Knopf. Recuperado de https://ehrafworldcultures.yale.edu/document?id=of07-002

Crittenden, A.N. y Blurton Jones, N.G. (2019). Culture Summary: Hadza. Human Relations Area Files. Recuperado de https://ehrafworldcultures.yale.edu/document?id=fn11-000

Darwin, C. (2010). El origen de las especies. Barcelona: Edaf. 
Diaz Cordova, D. (2011). Algunas reflexiones en torno al concepto de cultura. Manuscrito.

Durkheim, É. (2005). Las reglas del método sociológico. Biblioteca Nueva. Madrid: Biblioteca Nueva. Recuperado de http://www.digitaliapublishing.com/a/5181

Engels, F. (2017). La dialéctica de la naturaleza. Madrid: Akal.

Erdland, A. y Neuse, R. (1914). Marshall Islanders: Life And Customs, Thought And Religion Of A South Seas People. En Anthropos Bibliothek Ethnological Monographs (Vol. 2, p. HRAF ms: iii, 296 [original: xiv, 376 ]). [s.n.]. Recuperado de https://ehrafworldcultures.yale. edu/document?id=or11-003

Fourie, L. (1928). Bushmen of South West Africa. Cape Times limited. Recuperado de https://ehrafworldcultures.yale.edu/document?id=fx10-008

Gould, S.J. (2002). The structure of evolutionary theory. Belknap Press of Harvard University Press.

Gullick, C.J.M.R. (1976). Exiled From St. Vincent: The Development Of Black Carib Culture In Central America Up To 1945. Progress Press. Recuperado de https:// ehrafworldcultures.yale.edu/document?id=sa12-003

Hansen, H.H. (1961). Kurdish Woman'S Life: Field Research in a Muslim Society, Iraq. National museet. Recuperadodehttps://ehrafworldcultures.yale.edu/document?id=ma11-004

Hegel, G.W.F. (2013). Ciencia de la lógica. Buenos Aires: Las Cuarenta.

Hirschon, R. (1989). Heirs Of The Greek Catastrophe: The Social Life Of Asia Minor Refugees In Piraeus. Clarendon Press; Oxford University Press. Recuperado de https://ehrafworldcultures.yale.edu/document?id=eh01-058

Hudson, A.E. (1938). Kazak Social Structure. Published for the Department of Anthropology Yale University, by the Yale University Press; H. Milford, Oxford University Press. Recuperado de https://ehrafworldcultures.yale.edu/document?id=rq02-002

Keeling, R. (1992). Cry For Luck: Sacred Song And Speech Among The Yurok, Hupa, and Karok Indians Of Northwestern California. University of California Press. Recuperado de https://ehrafworldcultures.yale.edu/document?id=ns31-018

Kenny, M.G. (2011). Culture Summary: Nyakyusa And Ngonde. Human Relations Area Files. Recuperado de https://ehrafworldcultures.yale.edu/document?id=fn17-000

Lessa, W.A. (1950). Ethnography Of Ulithi Atoll. University of California. Recuperado de https://ehrafworldcultures.yale.edu/document?id=or20-001

McGee, J.T. (1961). Cultural Stability and Change Among The Montagnais Indians Of The Lake Melville Region Of Labrador. Catholic University of America Press. Recuperado de https://ehrafworldcultures.yale.edu/document?id=nh06-026

Mead, M. (1930). Growing Up in New Guinea: A Comparative Study of Primitive Education. W. Morrow \& Company. Recuperado de https://ehrafworldcultures.yale.edu/ document?id=om06-001

Morin, E. (2009). Introducción al pensamiento complejo. Barcelona: Gedisa.

Murdock, G.P. (1967). Ethnographic Atlas: A Summary. Ethnology, 6(2), 109. Recuperado de https://doi.org/10.2307/3772751

Ohnuki-Tierney, E. (1974). Ainu Of The Northwest Coast Of Southern Sakhalin. Waveland Press, Inc. Recuperado de https://ehrafworldcultures.yale.edu/document?id=ab06-012 
OMS | Lactancia materna. (Sin fecha). WHO. Recuperado de http://www.who.int/topics/ breastfeeding/es/

Pagés, G. y Scholl, B. (1933). Hamitic Kingdom In The Center Of Africa: In Ruanda On The Shores Of Lake Kivu (Belgian Congo). En Mémoires: Vol. 1 (p. HRAF ms: v, 293 [original: iv, 704, 29 plates]). Libraire Falk fils, Georges van Campenhout, Successeur. Recuperado de https://ehrafworldcultures.yale.edu/document?id=fo57-003

Prigogine, I. (1997). Las leyes del caos. Barcelona: Crítica.

Rappaport, R.A. (2000). Pigs for the ancestors: Ritual in the ecology of a New Guinea people (2. ed). Long Grove: Waveland Press.

Reynoso, C. (1998). Corrientes en antropología contemporánea (2. ed). Buenos Aires: Editorial Biblios.

Reynoso, C. (2009). Modelos o metáforas: Crítica del paradigma de la complejidad de Edgar Morin. Buenos Aires: SB ed.

Roa Bastos, A. (ed.) (2011). Las culturas condenadas. Asunción: Sevilibro.

Roberts, A. (2010). The incredible human journey. London: Bloomsbury.

Roscoe, J. (1861-1932). \& Mackie Ethnological Expedition to Central Africa, 1919-1920. (1923). Bakitara Or Banyoro: The First Part Of The Report Of The Mackie Ethnological Expedition To Central Africa. Cambridge: The University Press. Recuperado de https://ehrafworldcultures.yale.edu/document?id=fk11-014

Rosen, E. von. (1924). Ethnographical Research Work During The Swedish Chaco-Cordillera Expedition, 1901-1902. C. E. Fritze, Ltd. Recuperado de https://ehrafworldcultures. yale.edu/document?id=sk06-001

Samaja, J. y Galende, E. (2009). Epistemología de la salud reproducción social, subjetividad y transdisciplina. Buenos Aires: Lugar Editorial.

Titiev, M. (1951). Araucanian Culture in Transition. University of Michigan Press. Recuperado de https://ehrafworldcultures.yale.edu/document?id=sg04-001

Turnbull, C.M. (1965). Mbuti Pygmies: An Ethnographic Survey. American Museum of Natural History. Recuperado de https://ehrafworldcultures.yale.edu/document?id=fo04-001

Ungar, P.S. (2006). Evolution of The Human Diet: The Known, The Unknown, and The Unknowable: The Known, The Unknown, and The Unknowable. Oxford University Press, USA. Recuperado de http://www.myilibrary.com?id=223512

Warren, M. (2018). Mum's a Neanderthal, Dad's a Denisovan: First discovery of an ancienthuman hybrid. Nature, 560(7719), 417-418. Recuperado de https://doi.org/10.1038/ d41586-018-06004-0

Whiting, J.W.M. (1941). Becoming A Kwoma: Teaching And Learning In A New Guinea Tribe. Published for the Institute of Human Relations by Yale University Press. Recuperado de https://ehrafworldcultures.yale.edu/document?id=oj13-001

Wilbert, J. (1976). To Become a Maker of Canoes: an Essay in Warao Enculturation: v. 37. UCLA Latin American Center Publications, University of California. Recuperado de https://ehrafworldcultures.yale.edu/document?id=ss18-021

Zingg, R.M.(1938). Report of The Mr. And Mrs. Henry Pfeiffer Expedition for Huichol Ethnography: The Huichols: Primitive Artists. G.E. Stechert and Company. Recuperado de https://ehrafworldcultures.yale.edu/document?id=nu19-001 